\title{
Óbitos neonatais de recém-nascidos de baixo peso ao nascer
}

Maria Aparecida Munhoz Gaíva ${ }^{1}$, Fernanda Sanches Peres Lopes², Silvana Margarida Benevides Ferreira ${ }^{3}$, Leandro Felipe Mufato ${ }^{4}$

\section{RESUMO}

Analisar as principais características dos recém-nascidos de baixo peso ao nascer que foram a óbito em Cuiabá, Mato Grosso. Estudo a partir de uma coorte composta de nascidos vivos com peso ao nascer de 500-2499 gramas que evoluíram ao óbito antes de completarem 28 dias de vida. Os dados foram obtidos do Sistema de Informação sobre Nascidos Vivos e Sistema de Informação sobre Mortalidade. Dos 3.404 recém-nascidos estudados, 238 evoluíram para o óbito (7,0\%). Prevaleceram os óbitos entre os do sexo masculino, com peso entre 500-999 gramas, Apgar $<7$ no primeiro e no quinto minuto e com presença de anomalia congênita $(p<0,001)$; com mães com idade menor que 20 anos $(p=0,033)$; que realizaram de uma a seis consultas no pré-natal $(p=<0,001)$. Os resultados mostraram que os óbitos neonatais se relacionaram com a qualidade da atenção obstétrica e neonatal, por se tratarem majoritariamente de mortes evitáveis.

Descritores: Recém-Nascido de Baixo Peso; Epidemiologia Descritiva; Mortalidade Infantil; Enfermagem Pediátrica.

\footnotetext{
${ }^{1}$ Enfermeira, Doutora em Enfermagem em Saúde Pública. Professora Associada da Universidade Federal de Mato Grosso. Cuiabá, MT, Brasil. E-mail: mamgaiva@yahoo.com.br.

2 Enfermeira. Professora Interina na Universidade Federal de Mato Grosso. Barra do Garças, MT, Brasil. E-mail: fernanda.sanchesperes@gmail.com.

${ }^{3}$ Enfermeira. Doutora em Ciências. Professor Titular da Universidade de Cuiabá. Cuiabá, MT, Brasil. E-mail: silvana benevides@ hotmail.com.

${ }^{4}$ Enfermeiro, Mestre em Enfermagem. Discente do Programa de Pós-Graduação em Enfermagem, nível Doutorado, da Universidade Federal de Mato Gross. Docente da Universidade do Estado de Mato Grosso. Cuiabá, MT, Brasil. E-mail: leandro.mufato@gmail.com.
} 


\section{INTRODUÇÃO}

Bebês com baixo peso ao nascer são vulneráveis ao risco de morte, especialmente a precoce, além de problemas de saúde, em comparação com recém-nascidos de peso adequado. Atualmente no Brasil, o baixo peso ao nascer (BPN) representa, isoladamente, o principal fator associado ao risco de óbito no período neonatal, estando presente em $65 \%$ dos óbitos ocorridos nos primeiros 28 dias de vida ${ }^{(1)}$. Dessa forma, melhoras na qualidade da atenção no pré-natal refletem na diminuição do índice de mortalidade neonatal, assim como investimentos dos serviços de saúde na qualidade da assistência ao parto(2).

A Organização Mundial de Saúde (OMS) define que todo bebê nascido com o peso inferior a 2.500 gramas é considerado recém-nascido de baixo peso ao nascer e ainda pode ser classificado como extremo baixo peso ( $\leq$ 999 gramas) ou muito baixo peso ( $\leq 1.499$ gramas) ${ }^{(3)}$.

O BPN pode estar relacionado tanto ao parto prematuro, nascimento antes de 37 semanas de gestação, como devido à restrição de crescimento intrauterino ou da relação entre ambas as situações. Estima-se que anualmente 15 a $20 \%$ de todos os nascimentos no mundo são de BPN ${ }^{(3)}$.

Os recém-nascidos de BPN têm sido objeto de vários estudos em países como a Colômbia, Índia, Indonésia, Taiwan e África, investigando não só os preditores da mortalidade neonatal, mas também os fatores de risco para o nascimento com baixo peso ${ }^{(4-6)}$. No contexto nacional, apesar dos altos índices de mortalidade neonatal entre os recém-nascidos de BPN, anda são poucos os estudos que analisam a mortalidade neste grupo de crianças $^{(7-9)}$, assim como em Cuiabá, capital de um Estado da região Centro- Oeste brasileira ${ }^{(10)}$.

Considerando que existem diferenças na mortalidade neonatal nas diversas regiões do país, e que as características maternas e dos recém-nascidos desempenham papel importante nas condições de nascimento e na sobrevivência do bebê e que a mortalidade neonatal representa $67,1 \%$ do total de mortes infantis em Cuiabá(11) ${ }^{1}$ justifica-se, pois, a realização deste estudo, que tem por objetivo analisar as principais características dos recém-nascidos de baixo peso ao nascer que foram a óbito em Cuiabá, Mato Grosso.

\section{MÉTODOS}

Estudo descritivo a partir de uma coorte de todos recém-nascidos de baixo peso ao nascer, residentes em Cuiabá/MT, Brasil, que evoluíram ao óbito no período neonatal, entre 01 de janeiro de 2011 e 31 de dezembro de 2014.

Os dados foram coletados da Declaração de Nascidos Vivos (DNV) e Declaração de Óbito (DO), disponibilizados pela Vigilância Epidemiológica da Secretaria Estadual de Saúde do Mato Grosso. Os dados foram organizados em dois bancos com as informações que foram extraídas de cada declaração (DNV e DO), respectivamente.

Em seguida, foi aplicada a técnica de linkage entre os dois bancos de dados. Essa técnica consiste na integração de informações de duas ou mais bases independentes, através de variáveis em comum. Tem sido amplamente utilizada pois favorece a recuperação de registros incompletos e inconsistentes contribuindo para a melhoria da qualidade das informações. O linkage determinístico foi feito através do software Excel ${ }^{\circledR}$, a partir do concatenamento dos campos-chaves: número da DNV; data de nascimento; sexo e idade materna. A integração 
dos bancos resultou em $96,5 \%$ de vinculação. Um novo banco de dados foi construído e as variáveis selecionadas e categorizadas de acordo com a DNV e DO.

No período estudado tivemos 3.477 nascidos vivos de baixo peso, sendo que no processo de seleção da amostra foram excluídos os RN com o peso ao nascer inferior a 500 gramas (69) e os anencéfalos (04), totalizando a amostra total de 3.404 neonatos de baixo peso nascidos vivos. Destes, 238 evoluíram para o óbito. Assim, foram incluídos na amostra os 3.166 recém-nascidos BPN sobreviventes e 238 recém-nascidos de BPN que foram a óbito no período neonatal.

As seguintes variáveis foram selecionadas para análise: do recém-nascido (peso ao nascer; sexo; Apgar no 10 e 5으 minuto e presença de anomalia), das mães (idade, cor/raça, situação conjugal e ocupação); da assistência (tipo de parto; tipo de gravidez; consulta de pré-natal; duração da gestação e local do nascimento). Foram, também, analisadas as causas básicas dos óbitos neonatais obtidas no Sistema de Informação de Mortalidade (SIM), que tem como referência a Classificação Internacional de Doenças-10a Revisão (CID 10).

Utilizou-se para o gerenciamento e análise dos dados o Programa Microsoft Excel ${ }^{\circledR} 2016$ e o software Statistical Package for the Social Sciences (SPSS) versão 17.0. Adotou-se o teste qui quadrado $\left(\chi^{2}\right)$ para comparações de proporções segundo a frequência relativa das variáveis relacionadas aos óbitos ao nível de significância de $5 \%(p<0,05)$ e para a análise das causas básicas de óbitos, frequência absolutas e relativas.

O projeto de pesquisa foi aprovado pelo Comitê de Ética em Pesquisa, sob o parecer no 1283.468/2015 em consonância com a Resolução CNS no 466/2012 do Conselho Nacional de Saúde.

\section{RESULTADOS}

Dos 3.404 recém-nascidos incluídos no estudo, 238 evoluíram para o óbito (7,0\%).

A taxa de mortalidade neonatal foi de 70 óbitos/1000 NV. O maior número de óbitos, 177 (74,4\%) ocorreu no período neonatal precoce (taxa de 52 óbitos/1000 NV). Destes, 73 (41,2\%) nas primeiras 24 horas após o parto e $61(25,6 \%)$ no período neonatal tardio (18 óbitos/1000 NV).

Verificou-se maior prevalência de óbitos (55,3\%) entre os neonatos BPN do sexo masculino, com peso entre $500-999$ gramas (49,2\%), Apgar < 7 no primeiro minuto $(84,9 \%)$ e no quinto minuto de vida $(56,7 \%)$ e com presença de anomalia congênita $(12,2 \%)$ ( $p<0,05 \%)$ quando comparados com aqueles que sobreviveram e essa diferença foi estatisticamente significante $(<0,05)$ (Tabela1).

Quanto às características das mães de recém-nascidos que vieram a óbito, verificou-se: maior predominância de óbitos entre as mães com idade < 20 anos (23,5\%) do que aquelas com idade $\geq 35$ anos (10,9\%) $p=0,033$. Ressalta-se que apesar da importância dos extremos de idade materna, o maior número de óbitos ocorreu entre os RN de mães com idade entre 20-34 (65,6\%) (Tabela 2).

Quanto as características assistenciais relativas à gestação e ao parto verificou-se que 101/42,4\% dos óbitos foram decorrentes de partos vaginais ao comparar com os sobreviventes ( $p=0,034)$, as mães com uma a seis consultas pré-natais obtiveram maiores proporções de óbitos em relação àquelas com $\geq 7$ consultas $(p<0,001)$ e tempo de gestação inferior a 37 semanas (93\%) foram mais predisponentes à mortalidade $(p<0,001)($ Tabelas 3$)$. 
Tabela 1: Características relacionadas aos recém-nascidos de baixo peso que foram a óbito e dos sobreviventes segundo as variáveis relacionadas ao recém-nascido. Cuiabá, MT, Brasil, 2011-2014.

\begin{tabular}{|c|c|c|c|c|c|c|c|}
\hline \multirow{3}{*}{ Características Neonatais } & \multicolumn{6}{|c|}{ Recém-nascidos de baixo peso } & \multirow{3}{*}{$\chi^{2}$ (p-valor) } \\
\hline & \multicolumn{2}{|c|}{ Óbitos } & \multicolumn{2}{|c|}{ Sobreviventes* } & \multicolumn{2}{|c|}{ Amostra total } & \\
\hline & n (238) & $\%$ & $n(3166)$ & $\%$ & n (3404) & $\%$ & \\
\hline \multicolumn{8}{|l|}{ Sexo* } \\
\hline Masculino & 131 & 55,3 & 1427 & 45,1 & 1558 & 45,8 & $9,24(0,002)$ \\
\hline Feminino & 106 & 44,7 & 1739 & 54,9 & 1845 & 54,2 & 1 \\
\hline \multicolumn{8}{|l|}{ Peso ao nascer (g) } \\
\hline 500 a 999 & 117 & 49,2 & 100 & 3,1 & 217 & 6,4 & $942,41(<0,001)$ \\
\hline 1.000 a 1.499 & 52 & 21,8 & 274 & 8,7 & 326 & 9,6 & $146,87(<0,001)$ \\
\hline 1.500 a 2.499 & 69 & 29,0 & 2792 & 88,2 & 2861 & 84,0 & 1 \\
\hline \multicolumn{8}{|l|}{ Apgar 10 minuto** } \\
\hline$\leq 7$ & 202 & 84,9 & 752 & 23,9 & 954 & 28,0 & $407,59(<0,001)$ \\
\hline$\geq 8$ a 10 & 36 & 15,1 & 2401 & 76,1 & 2437 & 72,0 & 1 \\
\hline \multicolumn{8}{|l|}{ Apgar 5o minuto** } \\
\hline$\leq 7$ & 135 & 56,7 & 237 & 7,5 & 372 & 11,0 & $548,81(<0,001)$ \\
\hline$\geq 8$ a 10 & 103 & 43,3 & 2917 & 92,5 & 3020 & 89,0 & 1 \\
\hline \multicolumn{8}{|l|}{ Presença de anomalia* } \\
\hline Sim & 29 & 12,2 & 52 & 1,6 & 81 & 2,4 & $106,02(<0,001)$ \\
\hline Não & 208 & 87,8 & 3103 & 98,4 & 3311 & 97,6 & 1 \\
\hline
\end{tabular}

Fontes: Sistema de Informação sobre Nascidos Vivos (SINASC) e Sistema de Informação sobre Mortalidade (SIM), Brasil.

Legenda:

* Sobreviventes: Apgar 10 minuto (13); Apgar 5o minuto (12); Presença de anomalia (11).

** Ignorados: Óbitos: sexo (1); presença anomalia (1)

Tabela 2: Características relacionadas aos recém-nascidos de baixo peso que foram a óbito e dos sobreviventes segundo as variáveis maternas. Cuiabá, MT, Brasil, 2011-2014.

\begin{tabular}{|c|c|c|c|c|c|c|c|}
\hline \multirow{3}{*}{ Características Maternas } & \multicolumn{6}{|c|}{ Recém-nascidos de baixo peso } & \multirow{3}{*}{$\chi^{2}$ (p-valor) } \\
\hline & \multicolumn{2}{|c|}{ Óbitos } & \multicolumn{2}{|c|}{ Sobreviventes* } & \multicolumn{2}{|c|}{ Amostra total } & \\
\hline & n (238) & $\%$ & $\mathrm{n}(3166)$ & $\%$ & N (3404) & $\%$ & \\
\hline \multicolumn{8}{|l|}{ Idade materna (anos) } \\
\hline$<20$ & 56 & 23,5 & 528 & 16,7 & 584 & 17,1 & $4,52(0,033)$ \\
\hline $20-34$ & 156 & 65,6 & 2226 & 70,3 & 2382 & 70,0 & $0,23(0,631)$ \\
\hline$\geq 35$ & 26 & 10,9 & 412 & 13,0 & 438 & 12,9 & 1 \\
\hline \multicolumn{8}{|l|}{ Cor/raça materna** } \\
\hline Preta & 12 & 5,1 & 184 & 5,9 & 196 & 5,8 & $1,35(0,717)$ \\
\hline Amarela & 1 & 0,4 & 19 & 0,6 & 20 & 0,6 & \\
\hline Branca & 51 & 21,4 & 750 & 23,9 & 801 & 23,7 & \\
\hline Parda & 174 & 73,1 & 2183 & 69,6 & 2357 & 69,9 & \\
\hline \multicolumn{8}{|l|}{ Situação conjugal materna** } \\
\hline Sem companheiro & 81 & 34,6 & 936 & 29,6 & 1017 & 30,0 & $2,60(0,107)$ \\
\hline Com companheiro & 153 & 65,4 & 2225 & 70,4 & 2378 & 70,0 & 1 \\
\hline \multicolumn{8}{|l|}{ Ocupação materna** } \\
\hline Atividade remunerada*** & 103 & 43,3 & 1502 & 47,7 & 1605 & 47,3 & $1,69(0,192)$ \\
\hline Atividade no lar & 135 & 56,7 & 1650 & 52,3 & 1785 & 52,7 & 1 \\
\hline
\end{tabular}

Fontes: Sistema de Informação sobre Nascidos Vivos (SINASC) e Sistema de Informação sobre Mortalidade (SIM), Brasil. Legenda:

* Sobreviventes: Cor/raça materna (30); situação conjugal (5); ocupação materna (14).

** Ignorados: Óbitos: situação conjugal (4)

*** Dentre as ocupações destacaram-se os profissionais de saúde $(6,0 \%)$ e professores $(5,0 \%)$

Dos 238 óbitos estudados, 196 (82,3\%) foram considerados evitáveis, por causas relacionadas às afecções originadas no período perinatal. Dentre as causas básicas de óbito destacaram-se feto e RN afetados por fatores maternos e por complicações da gravidez, trabalho de parto e parto $(68 / 34,7 \%)$, infecção específica do período perinatal $(55 / 28,0 \%)$ e transtornos respiratórios e cardiovasculares (43/22,0\%). As demais causas não claramente 
evitáveis, malformações congênitas, corresponderam a 42/17,6\% do total dos óbitos, com predomínio das malformações do aparelho circulatório (Tabela 4).

Tabela 3: Características relacionadas aos recém-nascidos de baixo peso que foram a óbito e dos sobreviventes segundo as variáveis assistenciais relativas a gestação e ao parto. Cuiabá, MT, Brasil, 2011-2014.

\begin{tabular}{|c|c|c|c|c|c|c|c|}
\hline \multirow{3}{*}{ Características Assistenciais } & \multicolumn{6}{|c|}{ Recém-nascidos de baixo peso } & \multirow{3}{*}{$\chi^{2}$ (p-valor) } \\
\hline & \multicolumn{2}{|c|}{ Óbitos } & \multicolumn{2}{|c|}{ Sobreviventes* } & \multicolumn{2}{|c|}{ Amostra total } & \\
\hline & n (238) & $\%$ & $n(3166)$ & $\%$ & $n(3404)$ & $\%$ & \\
\hline \multicolumn{8}{|l|}{ Tipo de parto } \\
\hline Vaginal & 101 & 42,4 & 1127 & 35,6 & 1228 & 36,1 & $4,49(0,034)$ \\
\hline Cesário & 137 & 57,6 & 2039 & 64,4 & 2176 & 63,9 & 1 \\
\hline \multicolumn{8}{|l|}{ Tipo de gravidez** } \\
\hline Múltipla & 42 & 17,6 & 560 & 17,7 & 602 & 17,7 & $0,00(0,979)$ \\
\hline Única & 196 & 82,4 & 2601 & 82,3 & 2797 & 82,3 & 1 \\
\hline \multicolumn{8}{|l|}{ Consulta pré-natal** } \\
\hline Nenhuma & 11 & 4,9 & 57 & 1,9 & 68 & 2,1 & $23,40(0,000)$ \\
\hline 1 a 6 & 138 & 61,9 & 1223 & 39,7 & 1361 & 41,2 & $49,22(<0,001)$ \\
\hline$\geq 7$ & 74 & 33,2 & 1798 & 58,4 & 1872 & 56,7 & 1 \\
\hline \multicolumn{8}{|l|}{ Duração da gestação (semanas)** } \\
\hline$<37$ & 214 & 93,0 & 1888 & 60,7 & 2102 & 63,0 & $95,80(<0,001)$ \\
\hline$\geq 37$ & 16 & 7,0 & 1220 & 39,3 & 1236 & 37,0 & 1 \\
\hline \multicolumn{8}{|l|}{ Local do nascimento } \\
\hline Hospital & 235 & 98,7 & 3141 & 99,2 & 3376 & 99,2 & $0,60(0,438)$ \\
\hline Outros & 3 & 1,3 & 25 & 0,8 & 28 & 0,8 & 1 \\
\hline
\end{tabular}

Fontes: Sistema de Informação sobre Nascidos Vivos (SINASC) e Sistema de Informação sobre Mortalidade (SIM), Brasil.

Legenda:

* Sobreviventes: Tipo de gravidez (5); consulta pré-natal (88); duração da gestação (58).

** Ignorados: Óbitos: consulta pré-natal (15) duração da gestação (8)

Tabela 4: Distribuição da causa básica do óbito dos recém-nascidos de baixo peso ao nascer. Cuiabá, MT, Brasil, 2011-2014.

\begin{tabular}{lcc}
\hline \multicolumn{1}{c}{ Causas básicas de óbito (CID X) } & $\mathbf{n}$ & $\mathbf{\%}$ \\
\hline Afecções originadas no período perinatal & $\mathbf{1 9 6}$ & $\mathbf{1 0 0}$ \\
Transtornos respiratórios e cardiovasculares específicos do período perinatal (P20- P29) & 43 & 22,0 \\
Transtornos hemorrágicos e hematológicos do feto e do recém-nascido (P50-P61) & 5 & 2,5 \\
Infecção específica do período perinatal (P35- P39) & 55 & 28,1 \\
Transtornos relacionados com a duração da gestação e crescimento fetal (P05-P08) & 11 & 5,6 \\
Feto e RN afetados por fatores maternos e por complicações da gravidez, trabalho de parto e parto (P00-P04) & 68 & 34,7 \\
Outros transtornos originados no período perinatal (P90-P96) & 9 & 4,6 \\
Transtornos endócrinos e metabólicos transitórios e específicos do feto e RN (P70-P74) & 3 \\
Traumatismo de parto (P10-P15) & 1,5 \\
Malformações congênitas, deformidades e anomalias cromossômicas & 2 \\
Malformações congênitas do aparelho circulatório (Q20-Q28) & $\mathbf{1 , 0}$ \\
Malformações congênitas do aparelho respiratório (Q30-Q34) & $\mathbf{1 0 0}$ \\
Outras malformações do aparelho digestivo (Q38-Q45) & 13 & 31,0 \\
Malformação congênita do aparelho urinário (Q60-Q64) & 4 & 9,5 \\
Malformações congênitas e deformidades aparelho osteomuscular (Q65-Q79) & 3 \\
Outras malformações congênitas (Q80-Q89) & 7,1 \\
Malformações do sistema nervoso (Q00-Q07) & 2 \\
\hline
\end{tabular}

Dentre as afecções originadas no período perinatal predominaram os seguintes agravos: septicemia, feto e recém-nascido afetados por ruptura prematura das membranas, doença da membrana hialina, feto e recémnascido afetados por transtornos maternos hipertensivos e infecção não específica. 


\section{DISCUSSÃO}

Nesta investigação buscou-se analisar as características das mães de recém-nascidos de baixo peso que foram a óbito, características destes recém-nascidos e dos sobreviventes, configurando-se como as características da mortalidade neonatal em Cuiabá, Mato Grosso, no período de 2011 a 2014.

Sobre as características dos recém-nascidos de baixo peso ao nascer, o presente estudo mostrou predomínio de mortes no período neonatal precoce, principalmente entre os recém-nascidos de extremo baixo peso (EBP), resultado coincidente com outros estudos realizados no país ${ }^{(7-8,12)}$. Também na coorte prospectiva com 627 nascidos vivos com idade gestacional de 23-31 semanas e peso ao nascer de 500-1499g, em 19 maternidades públicas de nove capitais na Região Nordeste do Brasil, a mortalidade neonatal nas primeiras 24 horas foi elevada $(9,4 \%)^{(9)}$.

A literatura tem evidenciado que essas mortes precoces são decorrentes de complicações da gravidez e do parto e estão associadas às condições antenatais, ao periparto e à inadequada atenção aos recém-nascidos no momento do parto ${ }^{(9,13)}$.

Estudo realizado em Burkina Faso concluiu que os fatores que aumentam o risco de morte na população de baixo peso podem ser combatidos com melhores práticas assistenciais em saúde e que atenção especial deve ser dada nos primeiros dias de vida, devido a vulnerabilidade do recém-nato ao ambiente externo ${ }^{(14)}$.

No que diz respeito às características biológicas dos recém-nascidos, as pesquisas nacionais têm destacado o BPN, especialmente o extremo baixo peso, como fator associado ao óbito neonatal(9,13), semelhante ao encontrado no presente estudo. A prematuridade e o baixo peso ao nascer foram os principais fatores associados à morte neonatal na Pesquisa Nascer no Brasil, sendo que os prematuros extremos e aqueles com extremo baixo peso ao nascer tiveram chance 200 a 300 vezes maior de morrer nos primeiros 28 dias de vida em relação aos recém-nascidos a termo e com peso ao nascer $\geq 2500 \mathrm{~g}^{(15)}$.

Coorte retrospectiva que avaliou a mortalidade neonatal em recém-nascidos de muito baixo peso $(<1500 \mathrm{~g})$, em hospital público do Rio de Janeiro, mostrou que a mortalidade foi maior dentre as crianças de menor peso $(500-749 \mathrm{~g})^{(8)}$. Estudo realizado na cidade de São Paulo reafirmou esses achados, mostrando que nos neonatos de extremo baixo peso $(<1000 \mathrm{~g})$ a probabilidade de morte foi 5,5 vezes maior que a do grupo com peso entre 1000 a 1499g. Os resultados mostraram ainda que não houve sobreviventes entre aqueles com peso inferior a $700 \mathrm{~g}^{(7)}$.

Outra característica biológica encontrada nos recém-nascidos de baixo peso que foram a óbito no presente estudo foi o sexo masculino, considerada variável preditiva do óbito neonatal nas crianças de baixo peso ao nascer, sendo que em pesquisa na Região Nordeste do Brasil, os recém-nascidos de muito baixo peso do sexo masculino, apresentaram risco três vezes maior de morte comparado com o feminino ${ }^{(9)}$. Um dos argumentos para esse destaque ao sexo masculino é o amadurecimento mais tardio do pulmão fetal, aumentando a incidência de agravos respiratórios, uma das principais causas de óbito neonatal ${ }^{(13)}$, e também que o sexo feminino tem melhor adaptação e resposta ao estresse oxidativo, o que influencia no crescimento e na sobrevida fetal ${ }^{(16)}$.

Quanto ao Apgar, os resultados deste estudo são consoantes com a associação relatada em outras pesquisas entre os valores baixos dos índices de Apgar no primeiro minuto, e em especial, no quinto minuto de vida, com a ocorrência de óbito neonatal, independentemente do peso de nascimento ${ }^{(13)}$. Igualmente, na coorte com 213 recém-nascidos com peso inferior 1500g, em região sul da cidade de São Paulo, o Apgar < 7 no quinto 
minuto associou-se ao óbito neonatal(7), assim como na coorte prospectiva com 627 nascidos vivos com idade gestacional de 23-31 semanas e peso ao nascer de 500-1499g, em nove capitais na Região Nordeste do Brasil, em que observou-se forte associação do escore de Apgar no 50 minuto $<7$ com o óbito nas primeiras 24 horas de vida ${ }^{(9)}$.

O índice de Apgar no primeiro minuto de vida reflete as condições de vitalidade do recém-nascido e está relacionado à qualidade da assistência no momento do nascimento. Por sua vez, índices baixos no quinto minuto de vida apontam para a não recuperação do índice baixo no primeiro minuto de vida, sinalizando que a gravidade do recém-nascido continuou elevada e poderia justificar o desfecho negativo ${ }^{(17)}$.

A associação entre malformações congênitas e o óbito neonatal tem sido demonstrada em estudos nacionais, independentemente do peso ao nascer ${ }^{(15,17)}$, achado este também encontrado neste estudo, com um percentual de $12,2 \%$ de malformações congênitas nas crianças de BPN. Pesquisa que investigou os fatores maternos e infantis associados à mortalidade neonatal em Cuiabá/MT, com dados secundários de nascimento e óbitos do ano de 2010, mostrou no modelo final que as crianças com anomalia congênita foram as que apresentaram maior chance de óbito no período neonatal (OR=17,78; IC95\%:6,68-47,27) ${ }^{(2)}$.

É indiscutível a importância das malformações congênitas nas pesquisas sobre mortalidade neonatal, já que são a segunda causa de óbitos infantis no país, respondendo por $30 \%$ das mortes neonatais em algumas cidades brasileiras $^{(18)}$. A despeito das anomalias ou malformações congênitas serem consideradas alterações morfológicas e/ou funcionais detectáveis ao nascer ${ }^{(18)}$, ressalta-se que sua identificação e diagnóstico podem não ser possíveis logo após o parto, já que algumas malformações necessitam de avaliação e diagnóstico posterior ${ }^{(19)}$, como é o caso de determinadas cardiopatias, o que pode levar à subnotificação na DNV. Assim, o uso desse dado disponibilizado no sistema de informação tem que ser visto com ressalvas.

No âmbito das características maternas, são apontadas como principais fatores de risco para a mortalidade neonatal a idade, condições desfavoráveis de vida, baixo grau de instrução materno e as mães sem companheiro(20-21), nesta investigação apenas a idade da mãe associou-se ao óbito dos recém-nascidos de baixo peso, não tendo sido analisadas variáveis como escolaridade e condições de vida maternas. Destaca-se ainda, que apesar dos maiores riscos para o óbito do neonato de baixo peso se situarem nas faixas etárias maternas extremas, mulheres muito jovens e aquelas com idade avançada ${ }^{(20)}$, no presente estudo, mais de $65 \%$ das mães dos neonatos que foram a óbito estavam na faixa etária entre 20-34 anos.

Quanto às características assistenciais relativas à gestação e ao parto, observou-se associação entre óbito neonatal em recém-nascidos de baixo peso e o inadequado acompanhamento pré-natal (menos de seis consultas), o que também foi observado em pesquisa desenvolvida no Rio de Janeiro ${ }^{(8)}$. Esse resultado já foi evidenciado em pesquisa com crianças de BPN realizada anteriormente em Cuiabá(10). Há que se destacar que no presente estudo, quase a totalidade $(90,0 \%)$ dos neonatos tinha menos de 37 semanas de idade gestacional, o que pode ter influenciado no número de consultas de pré-natal. Dentre as características assistenciais relacionadas ao parto, a idade gestacional menor que 37 semanas destacou-se, semelhantemente a outros resultados de estudos em que a prematuridade foi um importante fator de risco para o óbito neonatal(8,12).

A qualidade do acompanhamento da gestação é descrita na literatura como um importante fator de risco para a mortalidade neonatal, em especial as variáveis referentes à realização ou não do pré-natal, número de 
consultas, início precoce do acompanhamento e capacitação dos profissionais ${ }^{(20)}$. Além disso, o adequado acompanhamento pré-natal permite a identificação de intercorrências da gestação, prevenindo o nascimento de crianças de muito baixo peso e os óbitos neonatais ${ }^{(7)}$.

Revisão integrativa da literatura sobre mortalidade infantil e assistência pré-natal evidenciou que esta relação se refere ao número insuficiente de consultas ou à baixa qualidade do atendimento à gestante. Segundo as pesquisas analisadas nesta revisão, mesmo quando o número e a rotina de consultas pré-natal foram adequados, ocorreram óbitos evitáveis, reafirmando que não apenas a quantidade de consultas e a rotina são importantes, mas que a qualidade do atendimento na gestação necessita ser considerada ${ }^{(22)}$.

O aumento da cobertura e da qualidade nas intervenções no pré-natal, e intra e pós-natal poderia evitar $71 \%$ das mortes neonatais em todo o mundo ${ }^{(23)}$. No presente estudo não foi avaliada a qualidade do acompanhamento pré-natal, apenas se contabilizou o número de consultas, o que pode ser considerado uma limitação da pesquisa.

A pesquisa Nascer no Brasil, estudo de base hospitalar composto por puérperas e seus recém-nascidos, realizado no período de fevereiro de 2011 a outubro de 2012, com entrevistas e avaliação de prontuários, apontou problemas na qualidade da atenção relacionados ao processo assistencial no pré-natal e no parto, indicando qualidade não satisfatória da assistência ${ }^{(15)}$.

No que concerne às causas de óbitos, em Cuiabá/MT, o maior número de óbitos nos BPN teve como causa principal as afeç̧ões originadas no período perinatal (82\%), considerados óbitos evitáveis, tal qual o panorama de causas dos óbitos neonatais no país ${ }^{(1)}$, assim como em outra pesquisa já realizada na mesma cidade ${ }^{(24)}$. As mortes infantis evitáveis são preveníveis, total ou parcialmente, por ações efetivas dos serviços de saúde ${ }^{(25)}$.

O grande número de óbitos neonatais evitáveis constatados no presente estudo sinaliza a existência de problemas relacionados à assistência à gestação, parto/ nascimento e puerpério e a necessidade de melhorias nos serviços que atendem a clientela materno-infantil, bem como, investimentos na capacitação dos profissionais de saúde.

Ressalta-se a importância da identificação das causas de óbito no enfoque da evitabilidade para a construção de indicadores para melhorar a qualidade da atenção à saúde, para o planejamento de intervenções preventivas e assistenciais(24), bem como, para avaliar e monitorar a qualidade da atenção à saúde ${ }^{(25)}$.

Autores $^{(15)}$ defendem a necessidade de se dar maior visibilidade aos processos assistenciais e aos desfechos perinatais, bem como, a implementação nas maternidades brasileiras das boas práticas no trabalho de parto e parto, para prevenção dos óbitos neonatais evitáveis, e, consequentemente, redução da mortalidade infantil.

Entre as limitações deste estudo, destacam-se o viés de aferição pelo uso de dados secundários, a não inclusão de variáveis como renda familiar, aleitamento materno, consumo de cigarro, não disponíveis nos sistemas de informações utilizados. Além da não exploração nesta análise do número de perdas fetais anteriores, complexidade e natureza jurídica (privado/público) do hospital de nascimento da criança, dentre outras variáveis. Apesar destas limitações, os resultados possibilitaram conhecer o comportamento da mortalidade neonatal e o perfil das mães e dos recém-nascidos de baixo peso, oferecendo dados para o planejamento de ações para redução dos óbitos neonatais desses recém-nascidos, bem como, para subsidiar o planejamento e avaliação de políticas locais. 


\section{CONCLUSÃO}

Os resultados permitem concluir que a mortalidade neonatal nas primeiras 24 horas de vida é elevada, e ocorreu principalmente em crianças do sexo masculino, de extremo baixo peso, com idade gestacional $<37$ semanas e com baixa vitalidade ao nascer e tiveram como principais causas de óbito as afeç̧ões originadas no período perinatal, sendo consideradas mortes evitáveis. Por sua vez, as mães dos recém-nascidos que evoluíram para o óbito apresentaram idade principalmente entre 20-34 anos, realizaram menos de sete consultas de prénatal e tiveram parto cesárea.

Neste contexto, os achados encontrados evidenciam que os óbitos neonatais se relacionam com a qualidade da atenção obstétrica e neonatal ofertada pelos serviços de saúde e podem ser utilizados por gestores e profissionais da saúde como indicadores para monitorar e reduzir as taxas de mortalidade, bem como, para avaliar a efetividade de alguns programas dirigidos à população materno-infantil.

Diante dos resultados e reflexões apresentadas, entende-se como necessário mais investimentos em pesquisas que avaliem a qualidade da atenção pré-natal, as condições de assistência ao parto e os cuidados imediatos ao nascimento em nossa realidade.

\section{REFERÊNCIAS}

1. Ministério da Saúde, Secretaria de Vigilância em Saúde, Departamento de Análise de Situação de Saúde. Saúde Brasil 2011: uma análise da situação de saúde e a vigilância da saúde da mulher. Brasília: Editora do Ministério da Saúde, 2012.

2. Gaíva MAM, Fujimori E, Sato APS. Maternal and child risk factors associated with neonatal mortality. Texto contexto - enferm [Internet]. 2016 [acesso em: 19 set. 2018];25(4):e2290015. Disponível em: http://dx.doi.org/10.1590/0104-07072016002290015. 3. World Health Organization. Global nutrition targets 2025: childhood overweight policy brief. Geneva: World Health Organization; 2014 [acesso em: 19 set. 2018]. Disponível em:

http://www.who.int/nutrition/publications/globaltargets2025_policybrief_overweight/en/.

4. Estrada-Restrepo A, Restrepo-Mesa SL, Feria NCC, Santander FM. Factores maternos relacionados con el peso al nacer de recién nacidos a término, Colombia, 2002-2011. Cad Saude Publica [Internet]. 2016 [acesso em: 19 set. 2018];32(11):e00133215.

Disponível em: http://dx.doi.org/10.1590/0102-311x00133215.

5. Sutan R, Berkat S. Does cultural practice affects neonatal survival- a case control study among low birth weight babies in Aceh Province, Indonesia. BMC Pregnancy Childbirth [Internet]. 2014 [acesso em: 19 set. 2018];14:324. Disponível em:

https://doi.org/10.1186/1471-2393-14-342.

6. Ilunga PM, Mukuku O, Mawaw PM, Mutombo AM, Lubala TK, Shongo Ya Pongomba M, et al. Risk factors for low birth weight in Lubumbashi, Democratic Republic of the Congo. Médecine et Santé Tropicales [Internet]. 2016 [acesso em: 19 set. 2018];26(4):38690. Disponível em: https://doi.org/10.1684/mst.2016.0607.

7. Almeida MF, Alencar GP, Schoeps D, Novaes HMD, Camplbell O, Rodrigues LC. Sobrevida e fatores de risco para mortalidade neonatal em uma coorte de nascidos vivos de muito baixo peso ao nascer, na Região Sul do Município de São Paulo, Brasil. Cad Saude Publica [Internet]. 2011 [acesso em: 19 set. 2018];27(6):1088-98. Disponível em: https://dx.doi.org/10.1590/S0102311X2011000600006.

8. Cardoso RCA, Flores PVG, Viera CL, Bloch KV, Pinheiro RS, Fonseca SC et al. Infant mortality in a very low birth weight cohort from a public hospital in Rio de Janeiro, RJ, Brazil. Rev. Bras. Saude Mater. Infant. [Internet]. 2013 [acesso em: 19 set. 2018];13(3):23746. Disponível em: https://dx.doi.org/10.1590/S1519-38292013000300005.

9. Castro ECM, Leite AJM, Guinsburg R. Mortality in the first $24 \mathrm{~h}$ of very low birth weight preterm infants in the Northeast of Brazil. Rev Paul Pediatr (English Ed) [Internet]. 2016 [acesso em: 19 set. 2018];34(1):106-13. Disponível em:

https://doi.org/10.1016/i.rppede.2015.12.008.

10. Gaíva MAM, Fujimori E, Sato APS. Neonatal mortality in infants with low birth weigh. Rev Esc Enferm USP [Internet]. 2014 [acesso em: 19 set. 2018];48(5):778-85. Disponível em: https://doi.org/10.1590/\$0080-6234201400005000002.

11. Lima JC, Mingarelli AM, Segri NJ, Zavala AAZ, Takano OA. Estudo de base populacional sobre mortalidade infantil. Cien Saude Colet [Internet]. 2017 [acesso em: 19 set. 2018];22(3):931-39. Disponível em: https://doi.org/10.1590/141381232017223.12742016.

12. Souza VFHG, Cavalcante RS, Marques RV, Neves DC0, Lima FAZ, Azevedo VND. Fatores associados à mortalidade de recémnascidos de baixo peso. Revista Paraense de Medicina [Internet]. 2013 [acesso em: 19 set. 2018];27(3):29-36. Disponível em: 


\section{http://files.bvs.br/upload/S/0101-5907/2013/v27n3/a3867.pdf.}

13. Nascimento RM, Leite AJM, Almeida NMGS, Almeida PC, Silva CF. Determinantes da mortalidade neonatal: estudo casocontrole em Fortaleza, Ceará, Brasil. Cad Saude Publica [Internet]. 2012 [acesso em: 19 set. 2018];28(3):559-72. Disponível em: https://doi.org/10.1590/S0102-311X2012000300016.

14. Coulibaly A, Baguiya A, Millogo T, Meda IB, Koueta F, Kouanda S, et al. Predictors of mortality of low birth weight newborns during the neonatal period: A cohort study in two health districts of Burkina Faso. Int J Gynaecol Obstet [Internet]. 2016 [acesso em: 19 set. 2018];135 supl. 1:S89-S92. Disponível em: https://doi.org/10.1016/i.ijgo.2016.08.006.

15. Lansky S, Friche AAL, Silva AAM, Campos D, Bittencourt SDA, Carvalho ML, et al. Pesquisa Nascer no Brasil: perfil da mortalidade neonatal e avaliação da assistência à gestante e ao recém-nascido. Cad Saude Publica [Internet]. 2014 [acesso em: 19 set. 2018 ];30 supl. 1:S192-207. Disponível em: https://doi.org/10.1590/0102-311X00133213.

16. Clifton VL. Review: sex and the human placenta: mediating differential strategies of fetal growth and survival. Placenta [Internet]. 2010 [acesso em: 19 set. 2018];31(supl.):33-9. Disponível em: https://doi.org/10.1016/j.placenta.2009.11.010. 17. Oliveira AR, Junior JCL, Costa MFS. Perfil dos óbitos de recém-nascidos ocorridos na sala de parto de uma maternidade do Rio de Janeiro, 2010-2012. Epidemiol Serv Saude. 2013 [acesso em: 03 dez 2017];22(3):501-8. Disponível em: https://doi.org//10.5123/S1679-49742013000300015.

18. Laurenti R, Siqueira AAF, Jorge MHPM, Gotlieb SLD, Pimentel EC. A importância das anomalias congênitas ao nascer. Rev Bras Crescimento Desenvolv Hum [Internet]. 2014 [acesso em: 19 set. 2018];24(3):328-38. Disponível em:

http://pepsic.bvsalud.org/scielo.php?pid=S0104-12822014000300013\&script=sci arttext\&tlng=pt.

19. Gabriel GP, Chiquetto L, Morcillo AM, Ferreira MC, Bazan IGM, Daolio LD, et al. Avaliação das informações das Declarações de Nascidos Vivos do Sistema de Informação sobre Nascidos Vivos (Sinasc) em Campinas, São Paulo, 2009. Rev Paul Pediatr [Internet]. 2014 [acesso em: 19 set. 2018];32(3):183-8. Disponível em: https://doi.org/10.1590/0103-0582201432306.

20. Borba GG, Neves ET, Arrué AM, Silveira A, Zamberlan KC. Fatores associados à morbimortalidade neonatal: um estudo de revisão. Saúde (Santa Maria) [Internet]. 2014 [acesso em: 19 set. 2018];40(1):9-14. Disponível em:

https://doi.org/10.5902/223658347774.

21. Glinianaia SV, Ghosh R, Rankin J, Pearce MS, Parker L, Pless-Mulloli T. No improvement in socioeconomic inequalities in birthweight and preterm birth over four decades: a population-based cohort study. BMC Public Health [Internet]. 2013 [acesso em: 19 set. 2018];13:345. Disponível em: https://doi.org/10.1186/1471-2458-13-345.

22. Figueiredo PP, Lunardi Filho WD, Lunardi VL, Pimpão FD. Infant mortality and prenatal care: contributions of the clinic in the light of Canguilhem and Foucault. Rev Lat Am Enfermagem [Internet]. 2012 [acesso em: 19 set. 2018];20(1):201-10. Disponível em: https://doi.org/10.1590/S0104-11692012000100026.

23. Bhutta ZA, Das JK, Bahl R, Lawn JE, Salam RA, Paul VK, et al. Can available interventions end preventable deaths in mothers, newborn babies, and stillbirths, and at what cost? Lancet [Internet]. 2014 [acesso em: 19 set. 2018];384(9940):347-70. Disponível em: https://doi.org/10.1016/S0140-6736(14)60792-3.

24. Gaíva MAM, Fujimori E, Sato APS. Mortalidade neonatal: análise das causas evitáveis. Rev Enferm UERJ [Internet]. 2015 [acesso em: 19 set. 2018];23(2):247-53. Dispaonível em: https://doi.org/10.12957/reuerj.2015.5794.

25. Malta DC, Sardinha LMV, Moura L, Lansky S, Leal MC, Szwarcwald CL, et al. Atualização da lista de causas de mortes evitáveis por intervenções do Sistema Único de Saúde do Brasil. Epidemiol. Serv. Saúde [Internet]. 2010 [acesso em: 19 set. 2018];19(2):1736. Disponível em: http://ref.scielo.org/339vcp. 